\title{
Calculation of rotor losses in PM machines with retaining sleeves using transfer matrices
}

\author{
Jaime Renedo Anglada 1,2,*, Suleiman M. Sharkh ${ }^{2}$, and Michael A. Yuratich ${ }^{3}$ \\ ${ }^{1}$ European Organization for Nuclear Research (CERN), Geneva, Switzerland \\ ${ }^{2}$ Mechatronics Research Group, Mechanical Engineering, University of Southampton, \\ Southampton, U.K. \\ ${ }^{3}$ TSL Technology Ltd, One Ropley Business Park, Ropley, U.K. \\ *email: renedo.jaime @ieee.org
}

May 4, 2018

\begin{abstract}
Accurate calculation of rotor losses in PM synchronous machines can be critical because these losses tend to be very small relative to others in the machine. Numerical methods, such as finite element analysis (FEA), can now provide accurate estimates of these losses, but they, especially 3D FEA, can be time consuming. Analytical methods therefore remain very useful as quick tools for estimating losses at the early design stages. This paper presents a new improved analytical method for the calculations of rotor eddy current losses in PM machines using a reformulation of the current sheet model into transfer matrices to solve Helmholtz's diffusion equation. Such methodology reduces the complexity of the problem significantly, particularly in machines with retaining sleeves, and simplifies the numerical evaluation of the resulting equations. A high speed PM machine with a retaining sleeve is presented as a case study. The analytical results are verified using FEA.
\end{abstract}

\section{Introduction}

Permanent magnet synchronous machines are commonly attributed the advantage over induction and brushed DC machines of having virtually no rotor losses. This is by and large a nearly true statement in low speed machines of conventional designs with distributed windings and sinusoidal rotor and stator fluxes using sine filters to suppress PWM current ripple. However, this is not the case in common designs using fractional slot concentrated windings with significant asynchronous harmonics, which can produce significant rotor losses [1]. The losses are further intensified by high-speed, particularly when directly driven by a PWM inverter, without a sine filter under high electric loading conditions. Examples of applications where this may be the case include: liquid cooled highly loaded PWM driven motors developed for electric vehicles and submersible pumps; motors used in high-speed gas compressors and machines tools; and high speed turbo generators used in exhaust energy recovery electric turbo-compounding systems.

Rotor losses tend to be very small compared to other losses in the machine, but due to the usually poor cooling of the rotor they can cause excessive rotor heating, and even failure. For example, in a commercial $60 \mathrm{~kW}, 60 \mathrm{krpm}$ generator with limited rotor cooling through the shaft $180 \mathrm{~W}$ of rotor loss is tolerable, but if the loss is around 250 $300 \mathrm{~W}$, the rotor will overheat, which will lead to failure. A relatively small error in the calculation of these losses at the design stage can therefore be critical as it may result in designing a machine that may fail or discarding a feasible and maybe good design.

Rotor losses are primarily caused by eddy currents induced by asynchronous flux harmonics. But there can be also significant hysteresis losses in the magnets, which can be larger than eddy current losses at low frequencies [2]. The hysteresis losses can be particularly important in machines with ferrite PMs [1, 3, 4] but less significant in machines with NdFeB PMs [5]. However, the methodology presented in this paper deals only with eddy current rotor losses. 
During the last 20 years there have been significant advances in the development of both analytical and numerical techniques for calculating rotor losses [1]. Great insights have been gained through both theoretical and experimental work, which in some cases have aided the development of commercial products $[6,7,8,9,10,11]$. Finite element analysis (FEA) software is capable of accurately calculating losses taking into account PWM external circuits, 3dimensional effects and magnet segmentation that are difficult to handle analytically. But 3D FEA in particular is still time consuming, with a typical solution using a moderate mesh size taking several days to compute using a PC with a CORE i7 processor and 32 GB of memory. Two-dimensional models are quicker, but can still take several hours, which is still too long especially at the early design stage when the machine proportions are constantly revised in the light of rotor dynamics, losses, availability of materials. FEA is usually reserved for the final design refinement stages.

Therefore, at the early design stages, when answers are needed in minutes rather than hours, analytical methods remain indispensable. In addition to being quick, they provide an invaluable insight into the causes of the losses and their dependence on the machines design parameters and construction, thus aiding design decisions.

Previous studies have successfully calculated rotor losses in inner rotor PM machines using a current sheet model $[7,12,13,14,9,15,16]$. However, this approach has a major drawback, as the number of regions in the model increases, the complexity of the problem increases very significantly because it is necessary to invert a large matrix (proportional to the number of cylindrical layers) to solve the linear system of boundary conditions [14]. Furthermore, such a matrix can be badly scaled and in cylindrical coordinates some of terms are obtained by evaluating Bessel functions with large complex arguments, which is not always possible using common solvers.

In this paper we propose an analytical method also based on the current sheet model. However, instead of solving directly the linear system of boundary conditions we obtain a transfer matrix between adjacent layers. This implies that only matrices of order two need to be inverted. Transfer matrices are commonly used to represent the magnetic field of magnets in the optic approximation, i. e., thin lens model, for the beam dynamics in the context of particle accelerators [17]. A similar approach has been used in the past to obtain the eddy currents in geometries with several rectangular regions $[18,19,20]$. Kirtley, for example, apply a surface coefficient in a similar way but in cylindrical coordinates but assume that the eddy currents are resistance limited (solving Laplace's equation), which means that it is applicable for thin regions only [21].

We have generalised the concept of the transfer matrix to cylindrical coordinates for both conducting and nonconducting regions and formulated it in a systematic form to solve Helmholtz's diffusion equation. The methodology proposed in this paper can be used for any number of cylindrical regions, conducting or non-conducting, excited by a cylindrical current sheet at an arbitrary position. This approach can be used to analyse inner rotor PM machines, outer rotor PM machines and magnetic brakes with any number of cylindrical layers without increasing the complexity of the problem. Furthermore, the transfer matrix of each region provides a direct insight into the behaviour of each layer for a given frequency.

The machine studied in this paper is a high speed PM machine with a carbon fibre retaining sleeve. The eddy current distribution for each asynchronous harmonic can be readily calculated in every conducting region. To illustrate the usefulness of the proposed methodology we will show the influence of the sleeve conductivity on rotor losses. These results are verified using transient FEA and compared to the methods presented in [20, 21].

The paper starts with a description of the machine that is presented as a case study in section 2 . Next, the proposed methodology is presented in section 3. Section 4 shows the analytical calculation of the magnetic field distribution in the air-gap. The results are presented in section 5. Finally, the conclusions are drawn in section 6.

\section{Description of the Machine under Study}

Fig. 1 shows a quarter cross-section of a high speed PM machine, which requires a sleeve to hold the magnets, making the effective air-gap relatively large. The parameters of the machine are shown in Table 1. The slots of the machine shown in Fig. 1 have tooth tips, but as long as the tooth tips are not saturated, which is largely the case in these machines, the rectangular slot model remains valid [22, 23]. The rectangular slot model assumes a slot without tooth-tips, but with the same slot opening as the machine shown in Fig. 1. 


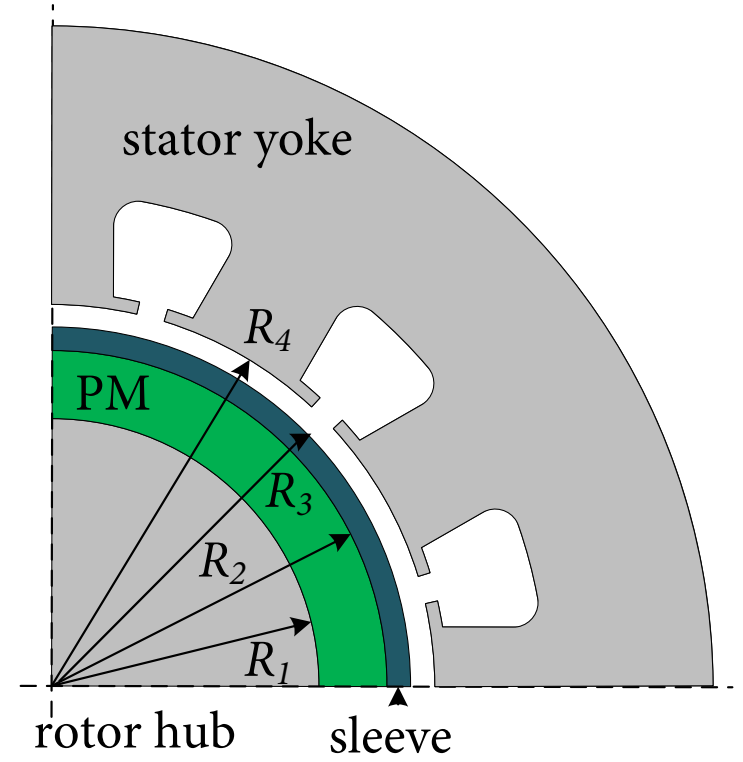

Figure 1: Quarter model of the PM synchronous machine under study.

Table 1: Parameters of the Machine

\begin{tabular}{lcc}
\hline Quantity & Symbol & Value \\
\hline Rated speed & $n_{r p m}$ & $65 \mathrm{krpm}$ \\
Rated power & $P_{n}$ & $50 \mathrm{~kW}$ \\
Rated current & $I_{n}$ & $60 \mathrm{~A}$ \\
Rated voltage & $V_{n}$ & $500 \mathrm{~V}$ \\
Number of poles & $2 p$ & 4 \\
Number of slots & $Q_{s}$ & 12 \\
Number of turns & $N_{t}$ & 7 \\
Core length & $L$ & $109 \mathrm{~mm}$ \\
Rotor hub radius & $R_{1}$ & $20 \mathrm{~mm}$ \\
Magnet outer radius & $R_{2}$ & $27.4 \mathrm{~mm}$ \\
Sleeve radius & $R_{3}$ & $30.35 \mathrm{~mm}$ \\
Stator radius & $R_{4}$ & $31.15 \mathrm{~mm}$ \\
Stator outer radius & $R_{5}$ & $54 \mathrm{~mm}$ \\
Stator permeability & $\mu_{r 5}$ & 5000 \\
Magnet's thickness & $h_{m}$ & $7.37 \mathrm{~mm}$ \\
Sleeve's thickness & $t_{s l}$ & $2.95 \mathrm{~mm}$ \\
Clearance gap & $h_{g}$ & $0.8 \mathrm{~mm}$ \\
Slot opening & $b_{o}$ & $3 \mathrm{~mm}$ \\
Rotor hub permeability & $\mu_{r 1}$ & 750 \\
Rotor hub conductivity & $\sigma_{r}$ & $6.7 \cdot 10^{6} \mathrm{~S} / \mathrm{m}$ \\
Magnet conductivity & $\sigma_{m}$ & $0.77 \cdot 10^{6} \mathrm{~S} / \mathrm{m}$ \\
Magnet material & - & $\mathrm{NdFeB}$ \\
Magnet permeability & $\mu_{r 2}$ & 1.07 \\
Magnet remanence & $B_{r}$ & $1.05 \mathrm{~T}$ \\
Magnet coercivity & $H_{c}$ & $781 \mathrm{kA} / \mathrm{m}$ \\
\hline
\end{tabular}


Taking into account that the permeability of the magnets and the sleeve is close to $\mu_{0}$ the effective air-gap length as defined in Fig. 1 is:

$$
g_{z}=h_{m}+t_{s l}+h_{g}=11.12 \mathrm{~mm},
$$

where $h_{m}$ is the magnet's thickness, $t_{s l}$ the sleeve's thickness and $h_{g}$ the clearance gap as shown in Table 1.

\section{Calculation of Rotor Losses using Transfer Matrices}

The proposed methodology assumes that the geometry is two-dimensional, therefore, it does not take into account end effects nor peripheral magnet segmentation. All the regions are assumed to be cylindrical, and accordingly circumferential segmentation is not taken into account. The properties of the materials are assumed to be linear and isotropic. Accordingly, saturation in the ferromagnetic elements is neglected and the anisotropic properties of the sleeve (if made of carbon fibre for example) have to be homogenized.

The underlying principle of the current sheet method is to represent each asynchronous field harmonic using a current sheet that generates a field harmonic of the same amplitude and space order $[12,14,15,16,23,24,25]$. The workflow of the process is as follows:

1. Calculate the asynchronous harmonics (at no-load or at on-load) present in the particular machine studied, this topic is described in detail in references [12, 14, 15, 16, 23, 24, 25];

2. Add the harmonics as vectors;

3. Represent each harmonic with a current sheet with the same space order and time order (assuming that PMs have no magnetization and that there are not currents in the windings); the amplitude of the current sheet is adjusted to produce the calculated magnetostatic flux density;

4. Calculate the rotor losses for each harmonic;

5. The total eddy current losses are the addition of all the harmonic losses by solving the diffusion equation.

Let us assume that we have a cylindrical geometry with $N$ regions, each of them with a conductivity $\sigma_{n}$ and a permeability $\mu_{n}$, as shown schematically in Fig. 2. The properties of the materials are assumed to be constant in each layer. There is a current sheet of time order $k$ and space order $q$ in between regions $h-1$ and $h$, which can be expressed as:

$$
J_{q k}=\hat{J}_{q k} \cos (q \theta+k \omega t)=\operatorname{Re}\left(\hat{J}_{q k} e^{j q \theta} e^{j k \omega t}\right),
$$

where $j=\sqrt{-1}$ is the imaginary unit.

Fig. 2 shows a simplified representation of the geometry of the problem. Only some of the regions are shown and the current sheet in between regions $h-1$ and $h$ is shown as a thick line.

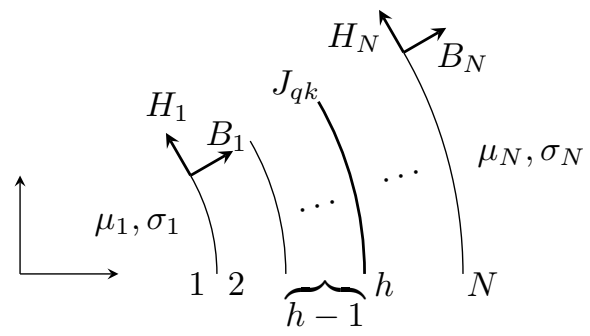

Figure 2: General model with current sheet on boundary $h$. 
The objective is to calculate the magnetic vector potential, $A$, in the each layer of the cylindrical domain. In cylindrical coordinates the Laplacian of $A$ assuming no variation in the $z$ direction is expressed as follows:

$$
\nabla^{2} A=\frac{1}{r} \frac{\partial}{\partial r}\left(r \frac{\partial A}{\partial r}\right)+\frac{1}{r^{2}} \frac{\partial^{2} A}{\partial \theta^{2}}
$$

Since the applied current sheet is sinusoidal in space and time, the magnetic vector potential, $A$, can be expressed as a phasor as

$$
A(\theta, r, t)=R(r) e^{j q \theta} e^{j k \omega t}
$$

accordingly, in steady state the Laplacian can be expressed as

$$
\nabla^{2} A=j k \omega \mu \sigma A
$$

Substituting (4) into (5) and re-arranging the terms we obtain

$$
\frac{d^{2} R(r)}{d r^{2}}+\frac{1}{r} \frac{d R(r)}{d r}-\left(j k \omega \mu \sigma+\frac{q^{2}}{r^{2}}\right) R(r)=0 .
$$

The general solution of this equation in a conductive region $(\sigma \neq 0)$ is:

$$
R(\kappa r)=C I_{q}(\kappa r)+D K_{q}(\kappa r),
$$

in which $\kappa^{2}=j k \omega \mu \sigma, C$ and $D$ are constants that are determined by applying the boundary conditions, and $I_{q}$ and $K_{q}$ are the modified Bessel functions of the first and second kinds of order $q$, respectively. The radial and tangential components of the magnetic field distribution are calculated as follows:

$$
\begin{aligned}
& B_{r}(\theta, r, t)=\frac{1}{r} \frac{\partial A}{\partial \theta}=\frac{1}{r} j q\left[C I_{q}(\kappa r)+D K_{q}(\kappa r)\right] e^{j q \theta} e^{j k \omega t}, \\
& B_{\theta}(\theta, r, t)=-\frac{\partial A}{\partial r}=-\kappa\left[C I_{q}^{\prime}(\kappa r)+D K_{q}^{\prime}(\kappa r)\right] e^{j q \theta} e^{j k \omega t} .
\end{aligned}
$$

However, for a non-conductive region $(\sigma=0)$ the solution of (6) is as follows:

$$
R(r)=C r^{q}+D r^{-q} \text {. }
$$

Therefore, the radial and tangential components of the magnetic field distribution are calculated as follows:

$$
\begin{aligned}
& B_{r}(\theta, r, t)=\frac{1}{r} \frac{\partial A}{\partial \theta}=\frac{1}{r} j q\left[C r^{q}+D r^{-q}\right] e^{j q \theta} e^{j k \omega t}, \\
& B_{\theta}(\theta, r, t)=-\frac{\partial A}{\partial r}=-\frac{q}{r}\left[C r^{q}-D r^{-q}\right] e^{j q \theta} e^{j k \omega t} .
\end{aligned}
$$

The only boundary condition is that there is no flux escaping the last layer, which is the same as saying that $B_{r}=0$ in layer $N$. At all the interfaces the radial component of the magnetic field, $B_{r}$, is continuous. The tangential field intensity, $H_{\theta}$, is continuous in all interfaces except layer $h$ in which there is a discontinuity by the amount of the current sheet value.

To reduce the complexity of the problem in the presence of several layers a transfer matrix approach is used to deal with the boundary conditions of the interfaces. The objective is to obtain a transfer matrix, $\left[\mathbf{T}_{n}\right]$, that relates the radial component of the magnetic field, $B_{r}$, and the tangential component of the field intensity, $H_{\theta}$, at the boundaries of region $n$. This can expressed as follows:

$$
\left[\begin{array}{l}
B_{n} \\
H_{n}
\end{array}\right]=\left[\mathbf{T}_{n}\right]\left[\begin{array}{l}
B_{n-1} \\
H_{n-1}
\end{array}\right]
$$


where $B_{n}$ is the radial component of the magnetic field at layer $n$ and $H_{n}$ is the tangential component of the field intensity at layer $n$. The transfer matrix can be obtained for each region based on the geometry and its properties. The calculation of this matrix for conductive regions and non-conductive regions is shown in sections 3.1 and 3.2, respectively.

Therefore, we can use the transfer matrix of each region to write the following equations:

$$
\begin{gathered}
{\left[\begin{array}{c}
B_{N} \\
H_{N}
\end{array}\right]=\left[\mathbf{T}_{N}\right]\left[\mathbf{T}_{N-1}\right] \ldots\left[\mathbf{T}_{h+1}\right]\left[\begin{array}{c}
B_{h} \\
H_{h}+\hat{J}_{q k}
\end{array}\right],} \\
{\left[\begin{array}{c}
B_{h} \\
H_{h}
\end{array}\right]=\left[\mathbf{T}_{h}\right]\left[\mathbf{T}_{h-1}\right] \ldots\left[\mathbf{T}_{2}\right]\left[\begin{array}{c}
B_{1} \\
H_{1}
\end{array}\right]}
\end{gathered}
$$

To solve this system it is necessary to apply the boundary condition, which is simply that $B_{N}=0$. Additionally, the magnetic field has to be finite at the origin. Therefore, we can write

$$
H_{1}=\beta_{1} B_{1}
$$

where

$$
\beta_{1}=\frac{j \kappa_{1} R_{1}}{\mu_{1} q} \frac{I_{q}^{\prime}\left(\kappa_{1} R_{1}\right)}{I_{q}\left(\kappa_{1} R_{1}\right)}
$$

when $\sigma_{1} \neq 0$ and

$$
\beta_{1}=\frac{j}{\mu_{1}}
$$

when $\sigma_{1}=0$.

Using the previous expression we can obtain the values of $B_{1}$ and $H_{N}$ as follows:

$$
\left[\begin{array}{c}
B_{1} \\
H_{N}
\end{array}\right]=[\mathbf{D}]^{-1}\left[\mathbf{T}_{N}\right]\left[\mathbf{T}_{N-1}\right] \ldots\left[\mathbf{T}_{h+1}\right]\left[\begin{array}{c}
0 \\
\hat{J}_{q k}
\end{array}\right]
$$

with

$$
[\mathbf{D}]=\left[\begin{array}{ll}
0 & 0 \\
0 & 1
\end{array}\right]-\left[\mathbf{T}_{N}\right]\left[\mathbf{T}_{N-1}\right] \ldots\left[\mathbf{T}_{2}\right]\left[\begin{array}{cc}
1 & 0 \\
\beta_{1} & 0
\end{array}\right]
$$

Once the values of $B_{1}$ and $H_{N}$ are known, they can be used to obtain all the rest using the transfer matrices, as shown in (14) an (15).

\subsection{Transfer matrix for a conductive region}

The transfer matrix, $\left[\mathbf{T}_{n}\right]$, directly relates the magnetic fields at the two boundaries of a given region $n$. Fig. 3 shows a schematic representation of a generic region. $H_{n-1}$ and $B_{n-1}$ are the tangential component of the field intensity and the radial component of the magnetic field at the inner boundary of region $n$, respectively.

The expression of the magnetic field distribution is shown in (8) and (9). Therefore, for a region $n$ :

$$
\left[\begin{array}{c}
B_{n-1} \\
H_{n-1}
\end{array}\right]=\left[\begin{array}{cc}
\frac{j q}{R_{n-1}} I_{q}\left(\kappa_{n} R_{n-1}\right) & \frac{j q}{R_{n-1}} K_{q}\left(\kappa_{n} R_{n-1}\right) \\
-\frac{\kappa_{n}}{\mu_{n}} I_{q}^{\prime}\left(\kappa_{n} R_{n-1}\right) & -\frac{\kappa_{n}}{\mu_{n}} K_{q}^{\prime}\left(\kappa_{n} R_{n-1}\right)
\end{array}\right]\left[\begin{array}{c}
C_{n} \\
D_{n}
\end{array}\right]
$$

therefore, re-arranging the terms:

$$
\left[\begin{array}{l}
C_{n} \\
D_{n}
\end{array}\right]=\frac{1}{F_{n}}\left[\mathbf{M}_{n}\right]\left[\begin{array}{l}
B_{n-1} \\
H_{n-1}
\end{array}\right]
$$




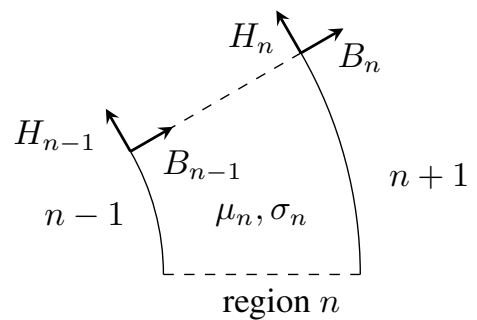

Figure 3: Simplified representation of a given region, $n$.

with

$$
\left[\mathbf{M}_{n}\right]=\left[\begin{array}{cc}
-\frac{\kappa_{n}}{\mu_{n}} K_{q}^{\prime}\left(\kappa_{n} R_{n-1}\right) & -\frac{j q}{R_{n-1}} K_{q}\left(\kappa_{n} R_{n-1}\right) \\
\frac{\kappa_{n}}{\mu_{n}} I_{q}^{\prime}\left(\kappa_{n} R_{n-1}\right) & \frac{j q}{R_{n-1}} I_{q}\left(\kappa_{n} R_{n-1}\right)
\end{array}\right]
$$

and

$$
F_{n}=\frac{j q \kappa_{n}}{\mu_{n} R_{n-1}}\left[K_{q}\left(\kappa_{n} R_{n-1}\right) I_{q}^{\prime}\left(\kappa_{n} R_{n-1}\right)-I_{q}\left(\kappa_{n} R_{n-1}\right) K_{q}^{\prime}\left(\kappa_{n} R_{n-1}\right)\right] .
$$

On the other hand we have that

$$
\left[\begin{array}{l}
B_{n} \\
H_{n}
\end{array}\right]=\left[\mathbf{N}_{n}\right]\left[\begin{array}{l}
C_{n} \\
D_{n}
\end{array}\right]
$$

with

$$
\left[\mathbf{N}_{n}\right]=\left[\begin{array}{cc}
\frac{j q}{R_{n}} I_{q}\left(\kappa_{n} R_{n}\right) & \frac{j q}{R_{n}} K_{q}\left(\kappa_{n} R_{n}\right) \\
-\frac{\kappa_{n}}{\mu_{n}} I_{q}^{\prime}\left(\kappa_{n} R_{n}\right) & -\frac{\kappa_{n}}{\mu_{n}} K_{q}^{\prime}\left(\kappa_{n} R_{n}\right)
\end{array}\right]
$$

Therefore, the transfer matrix is simply expressed as:

$$
\left[\mathbf{T}_{n}\right]=\frac{1}{F_{n}}\left[\mathbf{N}_{n}\right]\left[\mathbf{M}_{n}\right] .
$$

\subsection{Transfer matrix for a non-conductive region}

The methodology is exactly the same as in the case of a conductive region but the expression of the magnetic field distribution is shown in (11) and (12). Therefore, for a region $n$ :

$$
\left[\begin{array}{c}
B_{n-1} \\
H_{n-1}
\end{array}\right]=\left[\begin{array}{cc}
j q R_{n-1}^{q-1} & j q R_{n-1}^{-q-1} \\
-\frac{q}{\mu_{n}} R_{n-1}^{q-1} & \frac{q}{\mu_{n}} R_{n-1}^{-q-1}
\end{array}\right]\left[\begin{array}{c}
C_{n} \\
D_{n}
\end{array}\right],
$$

therefore, re-arranging the terms:

$$
\left[\begin{array}{c}
C_{n} \\
D_{n}
\end{array}\right]=\frac{1}{F_{n}}\left[\mathbf{M}_{n}\right]\left[\begin{array}{l}
B_{n-1} \\
H_{n-1}
\end{array}\right]
$$

with

$$
\left[\mathbf{M}_{n}\right]=\left[\begin{array}{cc}
\frac{q}{\mu_{n}} R_{n-1}^{-q-1} & -j q R_{n-1}^{-q-1} \\
\frac{q}{\mu_{n}} R_{n-1}^{q-1} & j q R_{n-1}^{q-1}
\end{array}\right]
$$


and

$$
F_{n}=\frac{2 j q^{2}}{\mu_{n} R_{n-1}^{2}} .
$$

On the other hand we have that

$$
\left[\begin{array}{l}
B_{n} \\
H_{n}
\end{array}\right]=\left[\mathbf{N}_{n}\right]\left[\begin{array}{l}
C_{n} \\
D_{n}
\end{array}\right]
$$

with

$$
\left[\mathbf{N}_{n}\right]=\left[\begin{array}{cc}
j q R_{n}^{q-1} & j q R_{n}^{-q-1} \\
-\frac{q}{\mu_{n}} R_{n}^{q-1} & \frac{q}{\mu_{n}} R_{n}^{-q-1}
\end{array}\right]
$$

Therefore, the transfer matrix is simply expressed as:

$$
\left[\mathbf{T}_{n}\right]=\frac{1}{F_{n}}\left[\mathbf{N}_{n}\right]\left[\mathbf{M}_{n}\right]
$$

\subsection{Calculation of losses}

Once the constants $C$ and $D$ are calculated for all the regions, the magnetic vector potential is known in all the domain and the currents induced in the axial direction are calculated as follows

$$
J_{\text {axial }}=-\sigma \frac{\partial A}{\partial t},
$$

where $\sigma$ is the conductivity of the material. The total rotor losses can be obtained by integrating $J_{a x i a l}^{2} / \sigma$ in all the domain or using the Poynting vector [23, 24, 9].

The amplitude of the current sheet that corresponds to each asynchronous harmonic, $\hat{J}_{q k}$, is effectively set to produce the same normal flux density on the surface of the magnet $\hat{B}_{q k}[14,23,24,16,26]$. In practice, the problem is solved by setting $\hat{J}_{q k}=1$ and calculating the corresponding losses $P_{q k 1}$ from the solution of the diffusion equation in the current sheet model. In addition, the Laplace equation (no eddy currents) is solved to find the corresponding $\hat{B}_{q k 1}$ when $\hat{J}_{q k}=1$. Finally, since the eddy current losses are proportional to the square of the field inducing them, the actual losses for a given $\hat{B}_{q k}$ are readily calculated as

$$
P_{q k}=\left(\frac{\hat{B}_{q k}}{\hat{B}_{q k 1}}\right)^{2} P_{q k 1} .
$$

In this paper, the value of $\hat{B}_{q k}$ is obtained using conformal mapping and the winding functions as shown in the following section.

\subsection{Comparison with the conventional approach}

The key step when obtaining the magnetic field distribution and eddy current distribution using our method is the solution of (19) and (20). In practice, this means inverting matrices of order 2.

On the other hand, in the conventional approach, to obtain all the unknown coefficients it is necessary to invert a matrix of order $(2 N-1)$, where $N$ is the number of regions [14, 15, 16]. Furthermore, in many cases the orders of magnitude of the elements of such a matrix are very different (due to very large differences in conductivity and permeability of the different materials), which gives rise to numerical convergence problems.

In terms of computational complexity, this can be expressed using the big $O$ notation [27, 28]. The mathematical complexity of inverting an order two matrix is $\mathcal{O}(1)$, because the number of operations carried out does not increase as the number of layers increases. But inverting an $n$ order matrix has a complexity of $\mathcal{O}\left(n^{3}\right)$ when using the GaussJordan elimination algorithm. 


\section{Magnetic Field Distribution in the Air-gap}

\subsection{No-Load Magnetic Field Distribution}

The no-load magnetic field distribution in the air-gap of the slotless configuration using the rotor's reference frame can be expressed using complex number notation as

$$
B_{s l}(\theta, r)=\sum_{n=1,3,5}^{\infty} K_{n}(r) \cos (n p \theta)+j \sum_{n=1,3,5}^{\infty} D_{n}(r) \sin (n p \theta),
$$

where the coefficients $K_{n}(r)$ and $D_{n}(r)$ are calculated according to [29]. The complex permeance (CP) function using the rotor's reference frame is

$$
\lambda(\theta, r, t)=\lambda_{a 0}+\sum_{m=1,2,3}^{\infty} \lambda_{a m}(r) \cos \left(m Q_{s}\left(\theta-\omega_{m} t\right)\right)+j \sum_{m=1,2,3}^{\infty} \lambda_{b m}(r) \sin \left(m Q_{s}\left(\theta-\omega_{m} t\right)\right),
$$

where the coefficients $\lambda_{a 0}, \lambda_{a m}(r)$ and $\lambda_{b m}(r)$ are calculated using conformal mapping [22, 25, 26] and $\omega_{m}=\omega / p$ is the mechanical speed of the rotor. Therefore, the magnetic field distribution of the slotted geometry is

$$
B_{n l}(\theta, r, t)=B_{s l}(\theta, r) \cdot \lambda^{*}(\theta, r, t)
$$

For the calculation of rotor losses we are interested in the amplitude of the asynchronous harmonics of the radial component of the magnetic field, as discussed in section 3. Therefore, combining (37), (38) and (39) we obtain:

$$
\begin{aligned}
\operatorname{Re}\left(B_{n l}(\theta, r, t)\right)= & \sum_{n=1,3,5}^{\infty} K_{n}(r) \cos (n p \theta) \times\left[\lambda_{a 0}+\sum_{m=1,2,3}^{\infty} \lambda_{a m}(r) \cos \left(m Q_{s}\left(\theta-\omega_{m} t\right)\right)\right] \\
& +\sum_{n=1,3,5}^{\infty} D_{n}(r) \sin (n p \theta) \times\left[\sum_{m=1,2,3}^{\infty} \lambda_{b m}(r) \sin \left(m Q_{s}\left(\theta-\omega_{m} t\right)\right)\right] .
\end{aligned}
$$

For a particular $m_{o}$, which means a time order $k=m_{o} Q_{s} / p$, we can re-arrange (40) to express explicitly each asynchronous harmonic. For each particular $n_{o}$ we have two asynchronous harmonics whose space order, $q$, and amplitude, $B_{q k}$, are calculated as follows:

$$
\begin{cases}q=k+n_{o}, & \text { and } B_{q k}=K_{n_{o}}(r) \frac{\lambda_{a m_{o}}}{2}-D_{n_{o}}(r) \frac{\lambda_{b m_{o}}}{2} \\ q=k-n_{o}, & \text { and } B_{q k}=K_{n_{o}}(r) \frac{\lambda_{a m_{o}}}{2}+D_{n_{o}}(r) \frac{\lambda_{b m_{o}}}{2}\end{cases}
$$

The machine studied in this paper has $Q_{s}=12$ slots and $p=2$ pole pairs. Therefore, the rotor frame harmonics are of time orders $6,12,18 \ldots$ Accordingly, the most important asynchronous harmonics are for $n=1$, which is the fundamental waveform of the slotless solution. This means that the most important asynchronous harmonics for time order 6 there exist space order 5 and 7; for time order 12 the asynchronous harmonics there exist space order 11 and 13; for time order 18 the asynchronous harmonics there exist space order 17 and 19; and so forth.

\subsection{Armature reaction flux harmonics}

The asynchronous harmonics in a symmetrical three-phase stator winding with $p$ pole pairs can be calculated using the well known equations shown in $[30,31]$. The amplitude of the armature reaction flux harmonics, $F_{q k}$, with space order $q$ and time order $k$ is calculated as follows:

$$
F_{q k}=\frac{3}{2} \frac{4}{\pi} \frac{N_{t}}{2 p} \frac{1}{q} K_{w q} \sqrt{2} I_{k}
$$


where $K_{w q}$ is the winding factor:

$$
K_{w q}=K_{d q} K_{p q} K_{s q}
$$

The expressions for the distribution factor, $K_{d q}$, the pitch factor, $K_{p q}$, and the skew factor, $K_{s q}$, can be found in [30]. In this paper we will consider a sinusoidal excitation, therefore all the harmonics are due to the fundamental harmonic, $I_{1}$.

The armature reaction $\mathrm{mmf}$ expressed in the rotor's reference frame is the following:

$$
F_{a r}=F_{1} \cos (\theta)+F_{5} \cos (6 \omega t+5 \theta)+F_{7} \cos (6 \omega t+7 \theta)+\cdots+F_{6 n \pm 1} \cos (6 n \omega t+(6 n \pm 1) \theta),
$$

where $n$ is an integer number. Therefore, the most significant asynchronous harmonics in this machine are: time order 6 with space orders 5 and 7, and time order 12 with space orders 11 and 13.

In the multilayer current sheet model each harmonic is represented as a current sheet at the stator bore $\left(R_{4}\right)$. The peak value of the current sheet can be calculated as follows [16]:

$$
\hat{J}_{q k}=\frac{p q}{R_{4}} F_{q k} .
$$

The amplitude of each harmonic produced for this current sheet is obtained by solving the Laplace equation in the cylindrical multilayer model:

$$
B_{q k}(r)=\hat{J}_{q k} R_{4} \mu_{0} \frac{R_{1}^{-2 q} r^{q}+r^{-q}}{r\left(R_{1}^{-2 q} R_{4}^{q}-R_{4}^{-q}\right)}
$$

\subsection{Resulting Magnetic Field}

The resulting magnetic field expressed as a complex number is simply the addition of the no-load and the stator's magnetic field distribution waveforms. It is important to note that for the calculation of the asynchronous harmonics the addition of the two fields has to take into account the phase of the harmonics [16]. However, in this paper we will assume that the phase angle is 0 for simplicity. This is the same as directly adding the amplitude of each asynchronous harmonic of the same time and space orders.

\section{Results and Discussion}

The proposed methodology has been used to calculate the rotor losses in the machine described in section 2, which can be modelled as 5 concentric cylindrical layers with a different conductivity and permeability. One of the aims of this section is to illustrate the importance of accurate calculation of eddy currents in the retaining sleeve, particularly as the conductivity of the sleeve increases. The results of two cases are presented in this section: Case 1: assuming that the conductivity of the sleeve is 0 (a theoretical insulating sleeve); and Case 2: the conductivity of the sleeve is $\sigma_{3}=2.2 \times 10^{4}[\mathrm{~S} / \mathrm{m}]$. Both cases are applied to the machine shown in Fig. 1 but assuming different values for the sleeve conductivity. The relative permeability of the sleeve for both cases is as assumed to be $\mu_{r}=1$.

Fig. 4 shows the radial component of the no-load magnetic field distribution at $r=R_{3}$ at an instant of time. It can be appreciated that there is a good agreement between the analytical solution obtained using the $\mathrm{CP}$ function described in section 4 and the results obtained using FEA.

The amplitude of the no-load asynchronous field harmonics obtained using the CP function is shown in table 2. The asynchronous harmonics can be obtained by doing a 2D Fourier Transform of the waveform of the radial component of the magnetic field distribution shown in Fig. 4 at several rotor positions or simply by evaluating (41).

The amplitude of the armature reaction asynchronous harmonics obtained according to section 4 is shown in table 3. 


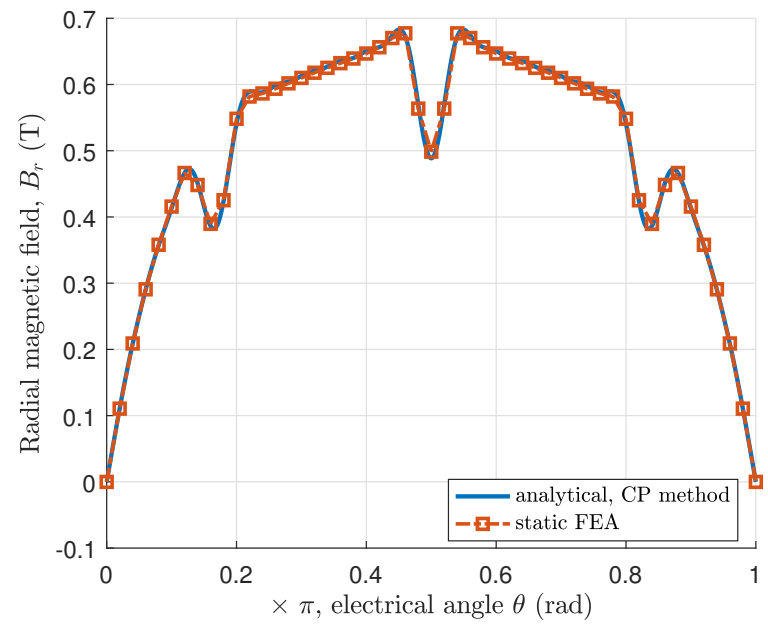

Figure 4: Magnetic field distribution in the air-gap at a particular rotor position $r=R_{3}$ obtained using static FEA and the $\mathrm{CP}$ function.

Table 2: No-load asynchronous harmonics obtained using the CP function, at $r=R_{2}+0.25 \mathrm{~mm}$.

\begin{tabular}{|c|ccc|}
\hline & \multicolumn{3}{|c|}{ Time order } \\
\hline Space order & 0 & 6 & 12 \\
\hline 1 & 832.28 & 0 & 0 \\
3 & 178.46 & 0 & 0 \\
5 & 90.49 & 5.40 & 0 \\
7 & 58.81 & 8.70 & 0 \\
9 & 43.07 & 2.44 & 0 \\
11 & 33.66 & 1.41 & 2.19 \\
13 & 27.35 & 0 & 3.53 \\
15 & 22.82 & 0 & 0 \\
\hline
\end{tabular}

Amplitude in (mT).

Table 3: Armature reaction asynchronous harmonics, at $r=R_{2}+0.25 \mathrm{~mm}$.

\begin{tabular}{|c|ccc|}
\hline & \multicolumn{3}{|c|}{ Time order } \\
\hline Space order & 0 & 6 & 12 \\
\hline 1 & 26.99 & 0 & 0 \\
3 & 0 & 0 & 0 \\
5 & 0 & -6.79 & 0 \\
7 & 0 & 4.21 & 0 \\
9 & 0 & 0 & 0 \\
11 & 0 & 0 & -1.62 \\
13 & 0 & 0 & 1.00 \\
15 & 0 & 0 & 0 \\
\hline
\end{tabular}

Amplitude in (mT). 
To verify the results obtained using the analytical method we have modelled the machine using FEA software. Fig. 5 shows the current density at an instant of time obtained using transient FEA.

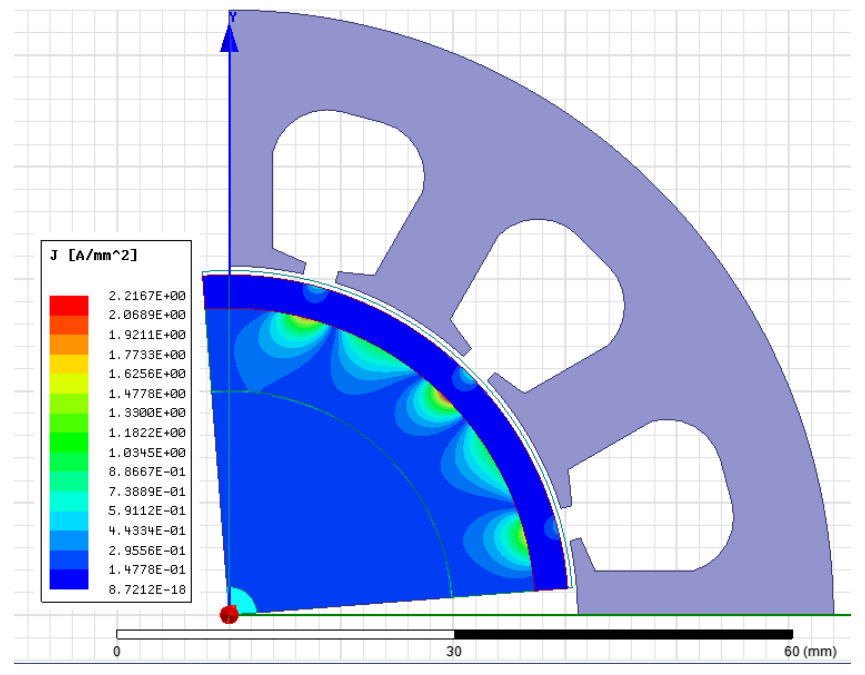

Figure 5: The distribution of $J_{z}$ in $\left(\mathrm{A} / \mathrm{mm}^{2}\right)$, obtained using transient FEA at no-load at nominal speed.

The total no-load and on-load rotor eddy current losses for Case 1 and Case 2 obtained using transient FEA are shown in Fig. 6. It can be appreciated that when the conductivity of the sleeve is not zero (Case 2) the total rotor losses increase.
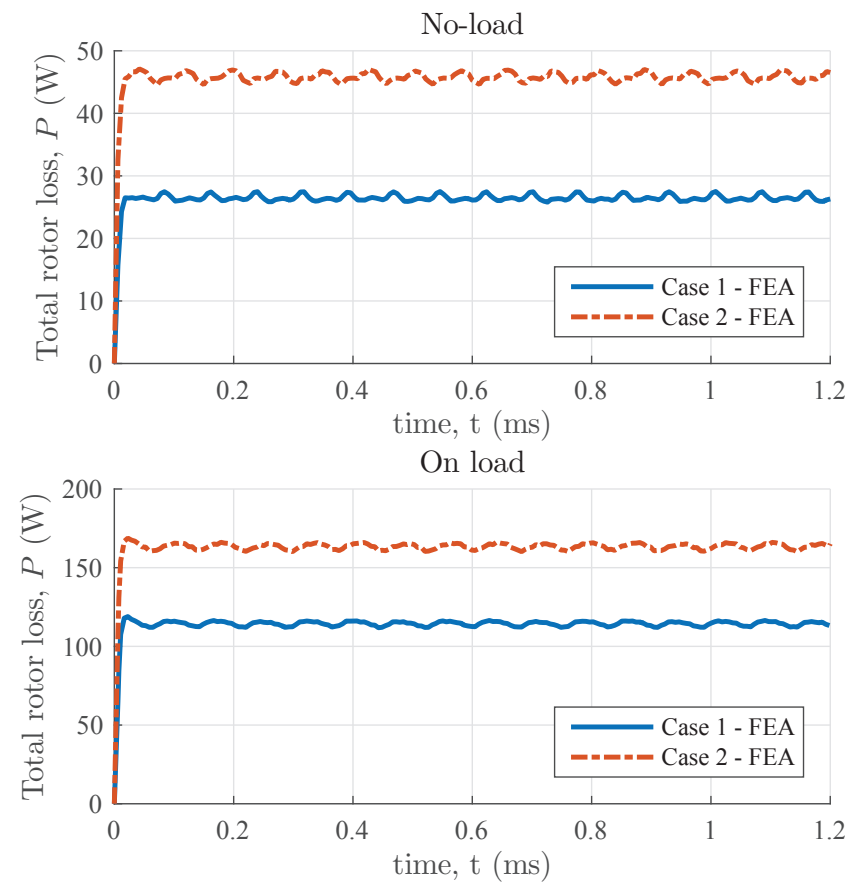

Figure 6: Total rotor losses obtained using transient FEA for Case $1\left(\sigma_{3}=0[\mathrm{~S} / \mathrm{m}]\right)$ and Case $2\left(\sigma_{3}=2.2 \times 10^{4}\right.$ $[\mathrm{S} / \mathrm{m}])$ at nominal speed.

The methodology proposed in section 3 has been implemented in a fairly recent dynamic programming language called Julia [32,33] and in Matlab. Julia has been developed recently at the MIT and it is targeted to scientific computing while trying to achieve high performance. All the relevant scripts and functions of this case study can be found in $[34,35]$. 
The total rotor on-load eddy current losses are shown in table 4. It can be appreciated that the results obtained with the proposed methodology present a reasonable level of accuracy for no-load and on-load conditions for Case 1 and for Case 2 compared with the results obtained using 2D transient FEA.

Table 4: Rotor losses obtained analytically $\left(P_{a}\right)$ and using transient FEA $\left(P_{F E A}\right)$ at nominal speed.

\begin{tabular}{|c|c|c|c|}
\hline \multicolumn{4}{|c|}{ No-load } \\
\hline & $\sigma_{3}(\mathrm{~S} / \mathrm{m})$ & $P_{a}(\mathrm{~W})$ & $P_{F E A}(\mathrm{~W})$ \\
\hline Case 1 & 0 & 24.79 & 26.49 \\
\hline Case 2 & $2.2 \times 10^{4}$ & 42.92 & 45.80 \\
\hline \multicolumn{4}{|c|}{ On-load } \\
\hline & $\sigma_{3}(\mathrm{~S} / \mathrm{m})$ & $P_{a}(\mathrm{~W})$ & $P_{F E A}(\mathrm{~W})$ \\
\hline Case 1 & 0 & 101.10 & 114.78 \\
\hline Case 2 & $2.2 \times 10^{4}$ & 150.93 & 163.44 \\
\hline
\end{tabular}

Figs. 7 and 8 show a comparison of the results obtained using transient FEA, the proposed analytical method using transfer matrices (TM), Freeman's rectangular method [20] and for the resistance limited model [8, 21]. Freeman's rectangular method does not provide very accurate results because the cylindrical regions are thick; this methodology is more suitable to study the losses in thin domains. The resistance limited model $[8,21]$ overestimates the losses for Case 1 because the skin depth for the relevant frequencies is larger than the PM's thickness. This model provides more accurate results for Case 2 because a large part of the losses is concentrated in the sleeve, in which the skin depth is larger. Finally, it can be appreciated that the proposed methodology using transfer matrices provides more accurate results in all operating conditions.

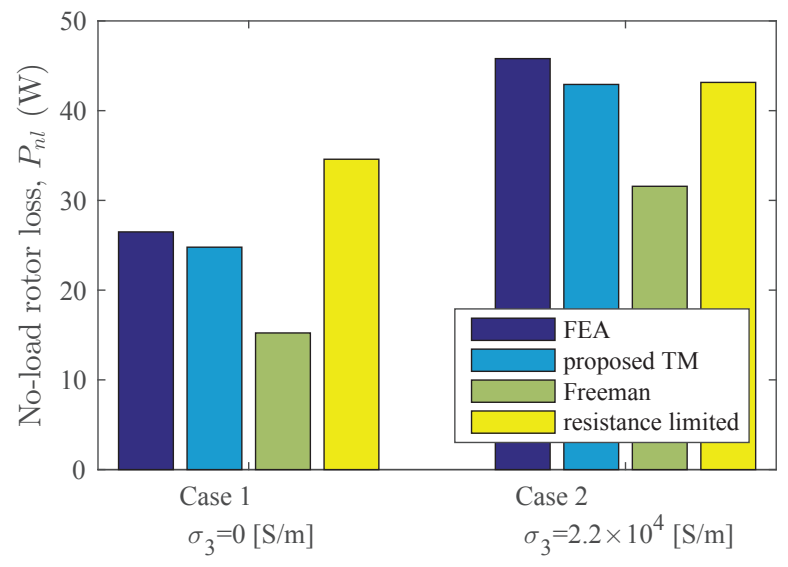

Figure 7: No-load rotor losses obtained using transient FEA, the proposed analytical method using transfer matrices (TM), Freeman's rectangular method [20] and for the resistance limited model [8, 21]; for Case $1\left(\sigma_{3}=0[\mathrm{~S} / \mathrm{m}]\right)$ and for Case $2\left(\sigma_{3}=2.2 \times 10^{4}[\mathrm{~S} / \mathrm{m}]\right)$ at nominal speed.

\section{Conclusions}

In this paper we have presented a novel re-formulation of the multi-layer current sheet model using transfer matrices to readily estimate the rotor eddy current losses by solving Helmholtz's diffusion equation in polar coordinates. The main advantage of the proposed methodology is that it allows the machine designer to deal with matrices of order two rather than large matrices, which reduces the mathematical complexity significantly and makes the calculations straight-forward.

The case study shows that the results obtained using the proposed methodology are consistent with static and 


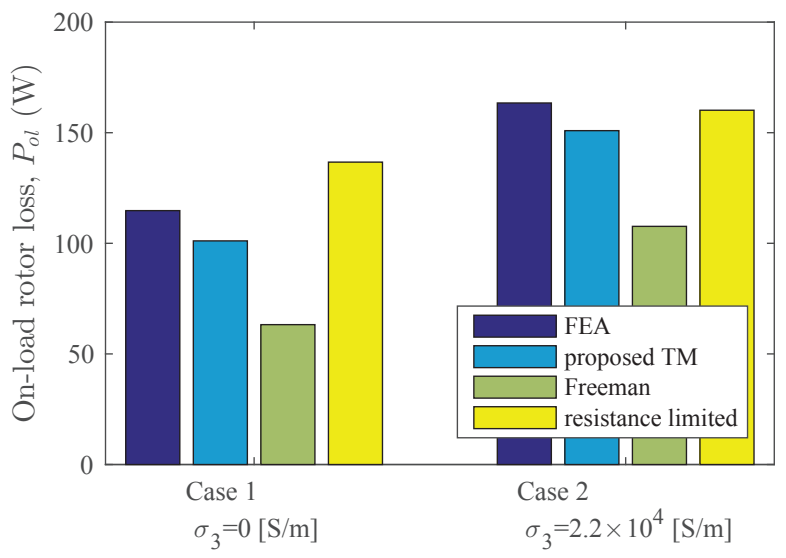

Figure 8: On-load rotor losses obtained using transient FEA, the proposed analytical method using transfer matrices (TM), Freeman's rectangular method [20] and for the resistance limited model $[8,21]$; for Case $1\left(\sigma_{3}=0[\mathrm{~S} / \mathrm{m}]\right)$ and for Case $2\left(\sigma_{3}=2.2 \times 10^{4}[\mathrm{~S} / \mathrm{m}]\right)$ at nominal speed.

transient FEA. Furthermore, the proposed methodology is more accurate than the rectangular approximation [20] and than the resistance limited model $[8,21]$.

While 2D-FEA provides a verification of the proposed transfer matrix method, more research is needed, using 3D-FEA and experimental measurement to assess the validity of the $2 \mathrm{D}$ current sheet method in general and determine the impact of $3 \mathrm{D}$ effects.

Finally, the simple formulation of the problem provides a great insight into the behaviour of the system. In particular, a simple inspection of the transfer matrix of one region can give an idea about how it will behave for a given frequency.

\section{Acknowledgment}

This work was supported by EPSRC and by TSL Technology Ltd.

\section{References}

[1] A. Tessarolo, "A survey of state-of-the-art methods to compute rotor eddy-current losses in synchronous permanent magnet machines," in 2017 IEEE Workshop on Electrical Machines Design, Control and Diagnosis (WEMDCD), April 2017, pp. 12-19.

[2] A. Fukuma, S. Kanazawa, D. Miyagi, and N. Takahashi, "Investigation of ac loss of permanent magnet of spm motor considering hysteresis and eddy-current losses," IEEE Transactions on Magnetics, vol. 41, no. 5, pp. 1964-1967, May 2005.

[3] I. Petrov, D. Egorov, J. Link, R. Stern, S. Ruoho, and J. Pyrhonen, "Hysteresis losses in different types of permanent magnets used in pmsms," IEEE Transactions on Industrial Electronics, vol. 64, no. 3, pp. 2502-2510, March 2017.

[4] D. Egorov, I. Petrov, J. Link, R. Stern, and J. J. Pyrhonen, "Model-based hysteresis loss assessment in pmsms with ferrite magnets," IEEE Transactions on Industrial Electronics, vol. 65, no. 1, pp. 179-188, Jan 2018.

[5] J. Pyrhonen, S. Ruoho, J. Nerg, M. Paju, S. Tuominen, H. Kankaanpa, R. Stern, A. Boglietti, and N. Uzhegov, "Hysteresis losses in sintered ndfeb permanent magnets in rotating electrical machines," IEEE Transactions on Industrial Electronics, vol. 62, no. 2, pp. 857-865, Feb 2015. 
[6] A. K. Nagarkatti, O. A. Mohammed, and N. A. Demerdash, "Special losses in rotors of electronically commutated brushless dc motors induced by non-uniformly rotating amature mmfs," IEEE Transactions on Power Apparatus and Systems, vol. PAS-101, no. 12, pp. 4502-4507, Dec 1982.

[7] O. Drubel and R. L. Stoll, "Comparison between analytical and numerical methods of calculating tooth ripple losses in salient pole synchronous machines," IEEE Transactions on Energy Conversion, vol. 16, no. 1, pp. 61-67, Mar 2001.

[8] K. Atallah, D. Howe, P. H. Mellor, and D. A. Stone, "Rotor loss in permanent-magnet brushless ac machines," IEEE Transactions on Industry Applications, vol. 36, no. 6, pp. 1612-1618, Nov 2000.

[9] Z. Q. Zhu, K. Ng, N. Schofield, and D. Howe, "Improved analytical modelling of rotor eddy current loss in brushless machines equipped with surface-mounted permanent magnets," IEE Proceedings - Electric Power Applications, vol. 151, no. 6, pp. 641-650, Nov 2004.

[10] N. Bianchi and E. Fornasiero, "Impact of mmf space harmonic on rotor losses in fractional-slot permanentmagnet machines," IEEE Transactions on Energy Conversion, vol. 24, no. 2, pp. 323-328, June 2009.

[11] X. Wu, R. Wrobel, P. H. Mellor, and C. Zhang, "A computationally efficient pm power loss mapping for brushless ac pm machines with surface-mounted pm rotor construction," IEEE Transactions on Industrial Electronics, vol. 62, no. 12, pp. 7391-7401, Dec 2015.

[12] P. J. Lawrenson, P. Reece, and M. C. Ralph, "Tooth-ripple losses in solid poles," Electrical Engineers, Proceedings of the Institution of, vol. 113, no. 4, pp. 657-662, April 1966.

[13] R. Stoll and J. Sykulski, "Modelling tooth ripple losses in the solid pole faces of synchronous machines," COMPEL - The international journal for computation and mathematics in electrical and electronic engineering, vol. 11, no. 1, pp. 105-108, 1992.

[14] N. T. Irenji, Calculation of Electromagnetic Rotor Losses in High-Speed Permanent Magnet Machines. PhD Thesis, University of Southampton, 1998.

[15] S. M. Sharkh, A. A. Qazalbash, N. T. Irenji, and R. G. Wills, "Effect of slot configuration and airgap and magnet thicknesses on rotor electromagnetic loss in surface pm synchronous machines," in International Conference on Electrical Machines and Systems (ICEMS), 2011, Aug 2011, pp. 1-6.

[16] A. A. Qazalbash, S. M. Sharkh, N. T. Irenji, R. G. Wills, and M. A. Abusara, "Rotor eddy loss in high-speed permanent magnet synchronous generators," IET Electric Power Applications, vol. 9, no. 5, pp. 370-376, 2015.

[17] S. Russenschuck, Field Computation for Accelerator Magnets. Wiley-VCH Verlag GmbH \& Co. KGaA, 2010.

[18] L. A. Pipes, "Matrix theory of skin effect in laminations," Journal of the Franklin Institute, vol. 262, no. 2, pp. $127-138,1956$.

[19] J. Greig and E. M. Freeman, “Travelling-wave problem in electrical machines," Electrical Engineers, Proceedings of the Institution of, vol. 114, no. 11, pp. 1681-1683, November 1967.

[20] E. M. Freeman, “Travelling waves in induction machines: input impedance and equivalent circuits," Electrical Engineers, Proceedings of the Institution of, vol. 115, no. 12, pp. 1772-1776, December 1968.

[21] J. L. Kirtley, "Surface coefficient for multipole magnetic field boundary condition problems," IEEE Transactions on Power Apparatus and Systems, vol. 94, no. 3, pp. 934-938, May 1975.

[22] D. Zarko, D. Ban, and T. A. Lipo, "Analytical calculation of magnetic field distribution in the slotted air gap of a surface permanent-magnet motor using complex relative air-gap permeance," IEEE Transactions on Magnetics, vol. 42, no. 7, pp. 1828-1837, July 2006.

[23] A. A. Qazalbash, S. M. Sharkh, N. T. Irenji, R. G. Wills, and M. A. Abusara, "Calculation of no-load rotor eddy-current power loss in pm synchronous machines," IEEE Transactions on Magnetics, vol. 50, no. 9, pp. 1-8, Sept 2014.

[24] _ "Rotor eddy current power loss in permanent magnet synchronous generators feeding uncontrolled rectifier loads," IEEE Transactions on Magnetics, vol. 50, no. 6, pp. 1-9, June 2014. 
[25] J. R. Anglada, S. M. Sharkh, and A. A. Qazalbash, "Analysis of slotting models for the calculation of no-load rotor losses in pm machines," in 2016 XXII International Conference on Electrical Machines (ICEM), Sept 2016, pp. 1325-1331.

[26] — " "Influence of curvature on air-gap magnetic field distribution and rotor losses in pm electric machines," COMPEL - The international journal for computation and mathematics in electrical and electronic engineering, vol. 36, no. 4, pp. 871-891, 2017. [Online]. Available: https://doi.org/10.1108/COMPEL-05-2016-0200

[27] P. Bachmann, Analytische Zahlentheorie. Leipzig: Teubner, 1894.

[28] J. V. Guttag, Introduction to Computation and Programming Using Python. The MIT Press, 2013.

[29] Z. Q. Zhu, D. Howe, and C. C. Chan, "Improved analytical model for predicting the magnetic field distribution in brushless permanent-magnet machines," IEEE Transactions on Magnetics, vol. 38, no. 1, pp. 229-238, Jan 2002.

[30] P. Alger, The nature of induction machines. Gordon and Breach, 1965.

[31] J. Pyrhonen, T. Jokinen, and V. Hrabovcova, Design of Rotating Electrical Machines. Hoboken, NJ: Wiley, 2009.

[32] J. Bezanson, S. Karpinski, V. B. Shah, and A. Edelman, "Julia: A fast dynamic language for technical computing," arXiv:1209.5145, 2012. [Online]. Available: https://arxiv.org/abs/1209.5145

[33] V. B. Shah, A. Edelman, S. Karpinski, J. Bezanson, and J. Kepner, "Novel algebras for advanced analytics in julia," in 2013 IEEE High Performance Extreme Computing Conference (HPEC), Sept 2013, pp. 1-4.

[34] J. R. Anglada, "Calculation of rotor losses using transfer matrices - matlab," https://github.com/jrenedo/TM_rotor_loss_matlab, 2018.

[35] — _ "Calculation of rotor losses using transfer matrices - julia," https://github.com/jrenedo/TM_rotor_loss_jl, 2018. 